\title{
Surtimiento de recetas a los afiliados al Seguro Popular de Salud de México
}

\author{
Francisco Garrido-Latorre, DSP, (1) Héctor Hernández-Llamas, D en Bioesta, ${ }^{(2)}$ \\ Octavio Gómez-Dantés, M en C. (3)
}

\section{Garrido-Latorre F, Hernández-Llamas H, Gómez-Dantés O. Surtimiento de recetas a los afiliados al Seguro Popular de Salud de México. Salud Publica Mex 2008;50 supl 4:S429-S436.}

\begin{abstract}
Resumen
Objetivo. Medir y comparar el porcentaje de recetas surtidas completamente a los usuarios de servicios ambulatorios y de los hospitales generales de los Servicios Estatales de Salud de México (SESA) afiliados y no afiliados al Seguro Popular de Salud (SPS) según condición de aseguramiento, además de medir la satisfacción de los usuarios de los SESA con el acceso a los medicamentos. Material y métodos. La información del estudio procede de cuatro encuestas de unidades ambulatorias y hospitalarias de los SESA que contaron con muestras probabilísticas de representatividad estatal. Las muestras de las unidades ambulatorias se seleccionaron mediante estratificación por nivel de atención y por condición de pertenencia a la red de servicios del SPS. Resultados. Los hallazgos indican que el porcentaje de recetas completamente surtidas ha mejorado en las unidades ambulatorias de los SESA, sobre todo en aquellas que ofrecen servicios a los afiliados al SPS y que alcanzan porcentajes de casi $90 \%$. Estos porcentajes, sin embargo, siguen siendo inferiores a los de las unidades ambulatorias de las instituciones de seguridad social. Los porcentajes de recetas surtidas en las unidades hospitalarias de los SESA también han mejorado, pero siguen siendo relativamente bajos. En casi todas las entidades federativas, conforme se ha incrementado el porcentaje de surtimiento completo de recetas, ha aumentado la satisfacción de los usuarios con el acceso a los medicamentos. Conclusiones. En 2006 , más de $50 \%$ de las entidades federativas presentaron altos niveles de surtimiento completo de recetas entre los afiliados al SPS ( $\geq 90 \%$ ). El mayor problema en este sentido se encuentra en los hospitales, ya que sólo $44 \%$ de
\end{abstract}

Garrido-Latorre F, Hernández-Llamas H, Gómez-Dantés O. Dispensing prescriptions to persons affiliated with Popular Health Insurance in Mexico.

Salud Publica Mex 2008;50 suppl 4:S429-S436.

Este artículo está basado en el informe Evaluación del surtimiento de medicamentos a la población afiliada al Seguro Popular de Salud, que forma parte del siguiente informe: Secretaría de Salud. Sistema de Protección Social en Salud. Evaluación de procesos. México, DF: Secretaría de Salud, 2006.

(I) Secretaría de Salud. México.

(2) Subsecretaría de Coordinación Metropolitana y Enlace Gubernamental. Gobierno del Distrito Federal. México.

(3) Instituto Carso de la Salud AC. México.

Fecha de recibido: 15 de octubre de 2007 - Fecha de aceptado: 5 de junio de 2008

Solicitud de sobretiros: Dr. Octavio Gómez Dantés. Genovevo de la O 103-A, Col. Santa María Ahuacatitlán. 62 I00, Cuernavaca, Morelos, México.

Correo electrónico: ocogomez@yahoo.com 
los usuarios que recibieron una prescripción en los hospitales de los SESA en 2006 obtuvieron el surtimiento completo de sus recetas. Este hallazgo obliga a revisar la política de medicamentos del SPS, que ha privilegiado la inclusión de claves de alta prescripción pero muy bajo costo unitario en los servicios ambulatorios, a expensas de medicamentos de costo más alto y mayor eficacia terapéutica en la atención hospitalaria, que son los de mayor impacto en la economía de los hogares.

Palabras clave: recetas médicas; calidad de la atención de salud; satisfacción del paciente; seguridad social; México low-cost medications at ambulatory services at the expense of higher cost and more therapeutically effective medications for hospital care, the latter having a greater impact on household budgets.

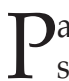
ara garantizar una buena atención médica es necesario contar oportunamente con una cantidad y una variedad adecuada de medicamentos, que además deben prescribirse sobre la base de evidencias científicas. La carencia de medicamentos en las unidades públicas de atención a la salud limita las opciones terapéuticas de los médicos, pone en riesgo la salud de los pacientes e impone a los usuarios cargas financieras que pueden llegar a convertirse en gastos catastróficos o empobrecedores.

El acceso a los medicamentos esenciales ha sido una preocupación añeja de las autoridades y los usuarios de los servicios públicos de salud en México y ha sido motivo de algunos trabajos de investigación. ${ }^{1-4}$

En enero de 2004 entraron en vigor en México las reformas a la Ley General de Salud que dieron origen al Sistema de Protección Social en Salud (SPSS) y a su brazo operativo, el Seguro Popular de Salud (SPS). ${ }^{5}$ Este seguro cubrirá en un lapso de siete años a todas aquellas personas que antes de la reforma no contaban con seguridad social, esto es, alrededor de 50 millones de mexicanos.

A diferencia de las instituciones de seguridad social [Instituto Mexicano del Seguro Social (IMSS), Instituto de Seguridad y Servicios Sociales de los Trabajadores del Estado (ISSSTE), Petróleos Mexicanos (PEMEX), Secretaría de Defensa (SEDENA) y Secretaría de Marina (SEMAR)], antes de la reforma de 2003 la Secretaría de Salud de México (SSA) no tenía la obligación de ofrecer medicamentos a los usuarios de sus servicios. La oferta de estos insumos era un compromiso solidario de los Servicios Estatales de Salud (SESA) que, en un sistema descentralizado como el mexicano, son las instancias responsables de la prestación de servicios de salud. A partir del 1 de enero de 2004, las unidades públicas de salud que ofrecen servicios a los afiliados al SPS tienen la obligación de entregar, sin desembolso en el momento de utilización de los servicios, los medicamentos necesarios para el tratamiento de todas aquellas condiciones de salud contempladas en el paquete de beneficios de este seguro, que se conoce como Catálogo Universal de Servicios Esenciales de Salud (CAUSES).

Los principales objetivos del estudio que dio origen a este artículo fueron los siguientes: a) medir y comparar el porcentaje de recetas surtidas completamente a los usuarios de servicios ambulatorios de los SESA según condición de aseguramiento; b) medir y comparar el porcentaje de recetas surtidas completamente a los usuarios de los hospitales generales de los SESA según condición de aseguramiento, y c) medir la satisfacción de los usuarios de los SESA con el acceso a los medicamentos.

Las principales hipótesis con las que se trabajó en este estudio fueron las siguientes:

1. La población afiliada al SPS presenta porcentajes de surtimiento completo de recetas mayores que la población sin seguridad social no afiliada al SPS en la atención ambulatoria y hospitalaria.

2. Las unidades de salud incorporadas a la red de servicios delSPS presentan un desempeño mejor en el surtimiento completo de recetas que las unidades no incorporadas al SPS.

3. Los SESA con buen desempeño en el surtimiento completo de recetas a los afiliados al SPS presentan también un buen desempeño en el surtimiento de recetas a la población no afiliada, bajo el supuesto de que han establecido arreglos gerenciales que facilitan la disponibilidad de medicamentos en las unidades de salud y los prestadores de servicios asumen una conducta "no discriminatoria".

Las encuestas que generaron los principales datos de este estudio se llevaron a cabo entre 2005 y 2006. A finales de 2005 el SPS operaba ya en las 32 entidades federativas y había alcanzado una cobertura de 11.4 millones de personas, cuya mayoría se ubicaba en los 
deciles I y II de ingreso. ${ }^{6}$ Los estados que mostraban mayores avances en la afiliación al SPS eran Aguascalientes, Colima y Tabasco, que tenían coberturas de afiliación a este seguro mayores de $60 \%$ de la población sin seguridad social.

Es importante señalar que para este estudio se contó con la colaboración de todas las autoridades de los SESA y que sus resultados se presentaron en el pleno del Consejo Nacional de Salud, al que asisten el secretario de salud federal y los secretarios de salud de las 32 entidades federativas. Esos resultados se utilizaron para ajustar la estrategia de mejora de surtimiento completo de recetas en la SSA.

\section{Material y métodos}

Los resultados que se presentan provienen de tres fuentes de datos: a) las versiones 2004 y 2005 de la Encuesta Nacional de Satisfacción y Trato Adecuado (ENSATA 2004 y 2005), contratada por la Dirección General de Evaluación del Desempeño de la SSA; b) las versiones 2005 y 2006 de la Medición Externa del Abasto de Medicamentos en los SESA (MEAM 2005 y 2006), contratada por la Dirección General de Planeación y Desarrollo (DGPlaDeS) de la SSA, ${ }^{7}$ y c) los registros del monitoreo interno del surtimiento de recetas, coordinado también por la DGPlaDeS.

La ENSATA 2004 es una encuesta de usuarios de servicios en unidades de atención ambulatoria del sector público basada en una muestra probabilística de unidades en una primera etapa y pacientes en una segunda. Cuenta con información de 88 unidades de atención ambulatoria distribuidas de la forma siguiente: $32 \mathrm{del}$ IMSS, 14 del ISSSTE, 31 de los SESA, y 11 del IMSSOportunidades. Los tamaños de muestra por institución fueron: 2251 en el IMSS, 964 en el ISSSTE, 2253 en los SESA y 540 del IMSS-Oportunidades, para un total de 6008 usuarios entrevistados. Los encuestadores pasaron de dos a tres días en cada unidad ambulatoria. La selección de los usuarios de los servicios de estas unidades varió en función de la demanda de atención. En las unidades rurales de los SESA y del IMSS-Oportunidades, en donde la demanda de atención es relativamente baja, se entrevistó a todos los usuarios. En las unidades urbanas se empleó un muestreo sistemático con arranque aleatorio. Los intervalos de muestreo variaron en función del número de usuarios por día. En general, se seleccionó un informante por cada dos o tres usuarios. A las unidades de atención no se les avisó de la llegada de los equipos de entrevistadores.

La ENSATA 2005 se realizó exclusivamente con pacientes entrevistados de una muestra de 74 hospitales de los SESA. En cada entidad federativa se seleccionaron de dos a tres hospitales para un total de 14484 usuarios, de los cuales 4844 (32.7\%) estaban afiliados al SPS.

Las MEAM 2005 y 2006 contaron con muestras probabilísticas con representatividad estatal. La muestra de unidades ambulatorias se seleccionó mediante estratificación por nivel de atención y por condición de pertenencia a la red de servicios del SPS. La primera MEAM se levantó en tres etapas. En cada etapa se visitaron 220 unidades y se entrevistó, en promedio, a 5342 usuarios de servicios, para un total de 16016 usuarios. La segunda se levantó en seis etapas. En cada etapa se visitaron 256 unidades y se entrevistó a 5342 usuarios.

En ambos tipos de encuestas se definió de la misma manera el surtimiento completo de recetas: consistencia total entre los medicamentos prescritos en la receta por el médico y los medicamentos recibidos por el paciente en la farmacia de la unidad. En todos los casos se pidió a los encuestadores que transcribieran, en un formato ad hoc, los nombres, presentación, dosis y duración del tratamiento (en días) de todos los medicamentos prescritos por el médico. Una vez anotada esta información, se verificaba si el o los medicamentos y las dosis entregados al usuario correspondían con lo señalado en la receta.

El monitoreo trimestral del surtimiento de recetas en las unidades de salud es una estrategia coordinada por la DGPlaDeS y forma parte de los indicadores de calidad (INDICA) promovidos en el marco de la Cruzada Nacional por la Calidad de los Servicios de Salud (CNCSS). Para conocer el nivel de surtimiento de recetas, las unidades de salud que voluntariamente se adhirieron a esta estrategia realizaban mediciones trimestrales mediante encuestas de usuarios seleccionados por el propio personal de las unidades. INDICA no define el número de usuarios entrevistados en sus encuestas en las entidades federativas que contribuían con información a este sistema. Debido a su baja confiabilidad, la información de este sistema se utiliza únicamente como referencia general y sólo se hace mención a ella en el primer párrafo de la sección de resultados. Cabe además señalar que, dado que el monitoreo del surtimiento de recetas en las unidades de salud incorporadas a la CNCSS no diferencia por condición de aseguramiento de los pacientes, sus resultados sólo proporcionan una imagen general del comportamiento del abasto de medicamentos en dichas unidades.

\section{Resultados}

\section{Surtimiento de recetas a usuarios de servicios de salud}

El sistema INDICA muestra un incremento progresivo del porcentaje de surtimiento completo de recetas en las 
unidades de los SESA. En 2003 los porcentajes llegaron a alcanzar cifras de apenas $60 \%$ para incrementarse a más de $80 \%$ en el último trimestre de 2004 y mantenerse estables hasta el tercer trimestre de 2006, con variaciones ligeras entre 80 y $83 \%$.

Las MEAM muestran un incremento de casi $6 \%$ en el surtimiento completo de recetas a los usuarios de los SESA afiliados al SPS entre 2005 y 2006, que pasa de 83.3 a $89.05 \%$. En la población no afiliada al SPS se observó un incremento todavía mayor en ese periodo (7.5\%). Sin embargo, la diferencia a favor de los asegurados por el SPS se mantuvo en alrededor de 11 puntos porcentuales (figura 1).

La información de la ENSATA 2005, que se levantó unos meses antes de la primera MEAM, indica que $80.6 \%$ de los usuarios de los servicios ambulatorios de los SESA afiliados al SPS surtían sus recetas completamente en las unidades de atención. En contraste, en la población no afiliada a este seguro este porcentaje ascendía a $61.2 \%$. Estos datos también muestran que el surtimiento completo de recetas en las unidades de atención ambulatoria de la seguridad social, en promedio, era 13 puntos porcentuales más alto que el observado en los usuarios del SPS (figura 2).

Los cambios observados en los SESA en las dos mediciones externas han sido notables: en 2006 casi se duplicó el número de entidades federativas que alcanzaron surtimientos completos de recetas por arriba de

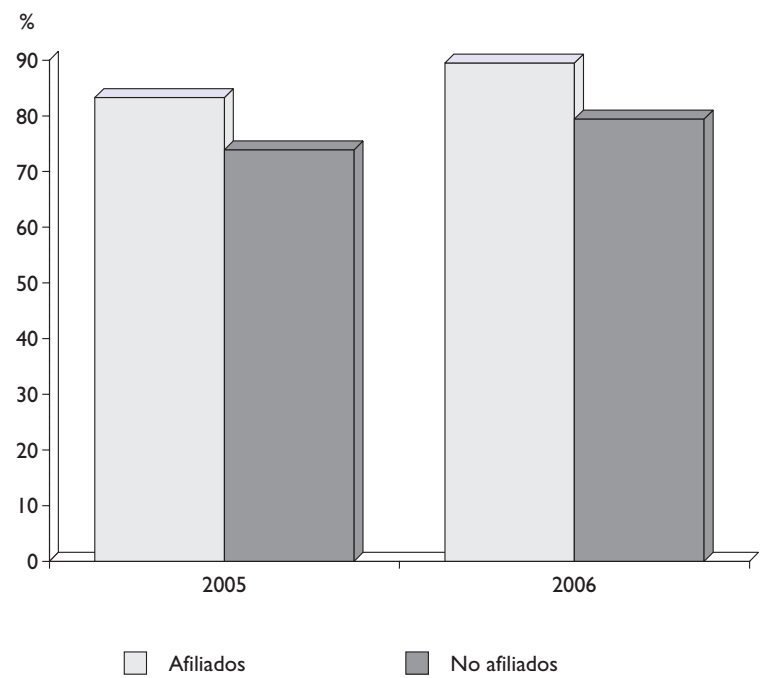

Figura I.Porcentaje de ReCETAS SURTidAs Completamente EN USUARIOS QUE RECIBIERON ATENCIÓN AMBULATORIA POR ESTATUS DE AFILIACIÓN AL SPS. MÉXICo 2005 Y 2006

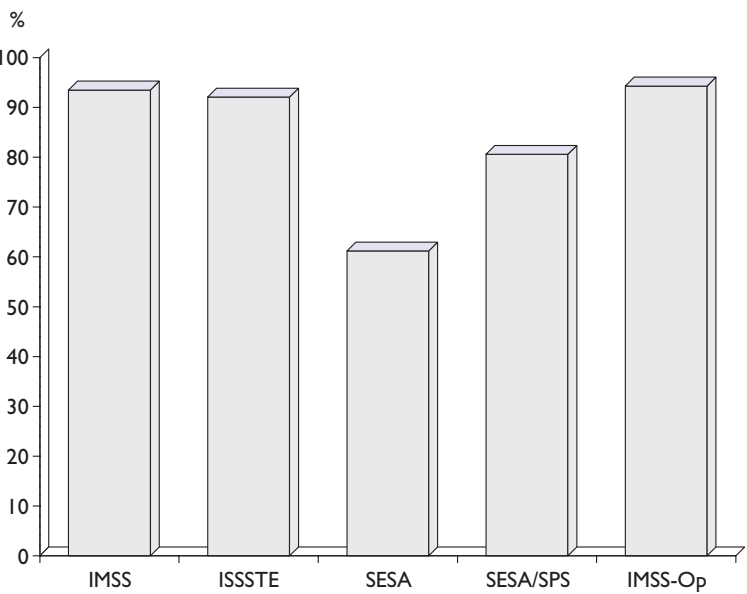

Figura 2. Porcentaje de ReCETAS SURTIDAs COMPLETAMENTE EN UNIDADES AMBULATORIAS POR INSTITUCIÓN.MÉXICO 2004

90\% respecto de 2005. De igual forma, mejoró el número de estados con surtimiento intermedio (80-89\%) y disminuyó la cifra de entidades con surtimientos muy bajos $(\leq 69 \%)$ (cuadro I).

Si la información se desagrega por estado, se observa que Tabasco es la entidad que mejor se desempeñó entre 2005 y 2006 en el surtimiento completo de recetas a los afiliados al SPS. En ambas mediciones sus cifras fueron superiores a 97\% (cuadro II). Baja California, Tlaxcala, Chihuahua, Tamaulipas y Campeche también mostraron desempeños altos en este rubro al mantener surtimientos completos por arriba de $90 \%$ en el mismo periodo.

Cabe destacar que 10 de las 12 entidades federativas que en 2005 presentaron un nivel de surtimiento completo de recetas bajo o muy bajo $(<79 \%)$, aumentaron considerablemente el acceso a los medicamentos por parte de los afiliados al SPS un año después. Destacan Hidalgo, Puebla, Aguascalientes y Veracruz, que en 2006 alcanzaron la categoría de alto surtimiento con cifras superiores a 90\% (cuadro II). Los incrementos alcanzados por estos estados variaron entre 28 y 36 puntos porcentuales. Zacatecas, Chiapas, Oaxaca, Michoacán y Durango lograron, en 2006, ubicarse en la categoría de desempeño intermedio (80-89\% de surtimiento) con incrementos de 9 a 29 puntos porcentuales respecto de sus cifras de 2005. Nayarit, a pesar de haber alcanzado un incremento de 17 puntos porcentuales, se mantuvo como una de las entidades con bajo surtimiento de recetas completas en 2006 (68\%). En contraste, Morelos y el Estado de México disminuyeron sus cifras en 2006 respecto de la medición realizada en 2005. 
Cuadro I

Niveles de surtimiento completo de Recetas EN UNIDADES AMBULATORIAS A POBLACIÓN AFILIADA AL SPS POR entidad federativa. MéXICo 2005-2006

\begin{tabular}{lcccc} 
& \multicolumn{5}{c}{ Número de entidades federales } \\
\cline { 2 - 6 } Porcentaje de surtimiento & 2005 & $\%$ & 2006 & $\%$ \\
$90-100$ & 9 & 29.0 & 16 & 50.0 \\
\hline $80-89$ & 10 & 32.3 & 12 & 37.5 \\
\hline $70-79$ & 7 & 22.6 & - & 0.0 \\
\hline$\leq 69$ & 5 & 16.1 & 4 & 12.5 \\
\hline Total & $31^{*}$ & 100 & 32 & 100
\end{tabular}

* En la medición 2005 el DF no tenía población afiliada al SPS SPS: Seguro Popular de Salud

Se esperaría que la población afiliada al SPS presentara, en todos los SESA, un porcentaje mayor de surtimiento de recetas completas en comparación con la población no afiliada. Sin embargo, este patrón no se cumple de manera uniforme. En 2006, en Chiapas, Nuevo León, Zacatecas y Querétaro, la población no afiliada al SPS presentó un surtimiento completo de recetas mayor que la población afiliada (cuadros II y III).

En poco más de un tercio de las entidades federativas se cumplió la hipótesis de que a mayor surtimiento de recetas en población afiliada al SPS, mayor surtimiento en población no afiliada. En este grupo se incluyen los estados de Aguascalientes, Baja California Sur, Campeche, Colima, Guanajuato, San Luis Potosí, Tabasco, Tamaulipas, Tlaxcala, Veracruz y Yucatán (surtimiento $290 \%$ ). En el mismo sentido, aunque con niveles de surtimiento inferiores (80-89\%), el surtimiento en ambas poblaciones fue similar en los estados de Coahuila, Distrito Federal, Guerrero, Oaxaca y Sinaloa. Finalmente, el Estado de México y Morelos presentaron niveles bajos de surtimiento de recetas tanto en la población afiliada al SPS como en la no afiliada a este seguro $(\leq 69 \%)$.

\section{Surtimiento de recetas en unidades ambulatorias incorporadas a la red de servicios del Seguro Popular de Salud}

Entre 2005 y 2006 también se observaron incrementos en el nivel general de surtimiento completo de recetas en las unidades incorporadas a la red de proveedores del SPS y en aquellas todavía no incorporadas (figura 3). En las primeras, el aumento relativo fue de $8.6 \%$ y en las segundas de $12.6 \%$. En ambos tipos de unidades, las mejoras observadas en 2006 ubican el surtimiento general de recetas en un nivel medio (80-89\%).

\section{Cuadro II}

Porcentaje de ReCetas surtidas Completamente EN UNIDADES AMBULATORIAS A POBLACIÓN AFILIADA AL SPS POR ENTIDAD FEderativa. MÉXICo 2005-2006

\begin{tabular}{lccc} 
Estado & 2005 & 2006 & Diferencia porcentual \\
Tabasco & 97.8 & 99.4 & 1.6 \\
\hline Yucatán & 90.6 & 99.1 & 9.4 \\
\hline Puebla & 70.0 & 98.8 & 41.1 \\
\hline Hidalgo & 61.4 & 97.8 & 59.3 \\
\hline Tlaxcala & 92.9 & 97.6 & 5.1 \\
\hline Veracruz & 73.7 & 97.4 & 32.2 \\
\hline Tamaulipas & 90.9 & 97.2 & 6.9 \\
\hline Quintana Roo & 81.0 & 96.6 & 19.3 \\
\hline Colima & 83.0 & 96.2 & 15.9 \\
\hline San Luis Potosí & 81.8 & 96.1 & 17.5 \\
\hline Campeche & 90.7 & 95.9 & 5.7 \\
\hline Baja California & 92.0 & 94.0 & 2.2 \\
\hline Guanajuato & 82.0 & 92.3 & 12.6 \\
\hline Chihuahua & 92.3 & 91.4 & -1.0 \\
\hline Aguascalientes & 67.5 & 91.4 & 35.4 \\
\hline Baja California Sur & 84.4 & 90.4 & 7.1 \\
\hline Durango & 76.6 & 89.5 & 16.8 \\
\hline Michoacán & 60.3 & 89.2 & 47.9 \\
\hline Chiapas & 75.4 & 88.9 & 17.9 \\
\hline Distrito Federa* & - & 88.5 & - \\
\hline Jalisco & 91.9 & 87.2 & -5.1 \\
\hline Coahuila & 89.4 & 87.1 & -2.6 \\
\hline Zacatecas & 77.2 & 86.2 & 11.7 \\
\hline Sonora & 81.4 & 85.0 & 4.4 \\
\hline Sinaloa & 88.5 & 81.4 & -8.0 \\
\hline Oaxaca & 65.7 & 81.0 & 23.3 \\
\hline Guerrero & 85.0 & 80.7 & -5.1 \\
\hline Nuevo León & 87.2 & 80.0 & -8.3 \\
\hline Nayarit & 58.1 & 68.0 & 17.0 \\
\hline Querétaro & 93.0 & 67.4 & -27.5 \\
\hline Morelos & 78.6 & 61.8 & -57.8 \\
\hline México & 30.1 & 89.05 & \\
\hline Total & & & \\
\hline
\end{tabular}

* En la medición 2005 el Distrito Federal no contaba con población afiliada al SPS

SPS: Seguro Popular de Salud

En 2006, 26 entidades federativas (81\%) presentaron un surtimiento completo de recetas en unidades incorporadas a la red de servicios del SPS medio o alto (80-100\%). Esta cifra en 2005 fue de alrededor de 50\%. Los estados que más se destacaron en este rubro, con porcentajes mayores de $97 \%$ de surtimiento completo, fueron Tabasco, Tlaxcala, Yucatán, Veracruz, Tamauli- 
Cuadro III

Porcentaje de ReCETAS SURTIDAS COMPLETAMENTE EN UNIDADES AMBULATORIAS A POBLACIÓN NO AFILIADA AL SPS POR ENTIDAD FEDERATIVA. MÉXICO 2005-2006

\begin{tabular}{lccc} 
Estado & 2005 & 2006 & Diferencia porcentual \\
Sinaloa & 96.3 & 86.7 & -9.6 \\
\hline Tabasco & 94.7 & 100 & 5.3 \\
\hline Yucatán & 93.6 & 100 & 6.4 \\
\hline Baja California & 92.6 & 89.8 & -2.8 \\
\hline Colima & 91.3 & 100 & 8.7 \\
\hline Tamaulipas & 85.7 & 98.7 & 13.0 \\
\hline Nuevo León & 84.4 & 98.6 & 14.2 \\
\hline Querétaro & 82.8 & 94.4 & 11.6 \\
\hline San Luis Potosí & 81.3 & 91.8 & 10.5 \\
\hline Chihuahua & 81.0 & 80.5 & -05 \\
\hline Tlaxcala & 80.9 & 94.8 & 13.9 \\
\hline Campeche & 79.5 & 98.7 & 19.2 \\
\hline Coahuila & 77.6 & 86.4 & 8.8 \\
\hline Zacatecas & 77.3 & 94.2 & 16.9 \\
\hline Puebla & 75.6 & 73.8 & -1.8 \\
\hline Guanajuato & 74.3 & 91.0 & 16.7 \\
\hline Quintana Roo & 73.8 & 81.3 & 7.5 \\
\hline Oaxaca & 72.8 & 84.3 & 11.5 \\
\hline Michoacán & 71.6 & 72.2 & 0.6 \\
\hline Morelos & 71.0 & 65.1 & -5.9 \\
\hline Veracruz & 70.8 & 96.4 & 25.6 \\
\hline Chiapas & 69.7 & 94.6 & 24.9 \\
\hline Nayarit & 67.7 & 89.2 & 21.5 \\
\hline México & 65.2 & 49.1 & -16.1 \\
\hline Durango & 64.4 & 74.9 & 10.5 \\
\hline Distrito Federal & 63.8 & 85.3 & 21.5 \\
\hline Guerrero & 63.5 & 80.2 & 16.7 \\
\hline Sonora & 62.9 & 56.8 & -6.1 \\
\hline Aguasaliscalientes & 57.6 & 92.0 & 34.4 \\
\hline Hidalgo & 56.7 & 76.9 & 20.2 \\
\hline Tajalifornia Sur & 34.0 & 100.0 & 77.6 \\
\hline
\end{tabular}

SPS: Seguro Popular de Salud

pas, Hidalgo, y Colima. Por el contrario, los estados de Guerrero, Morelos y México se ubicaron en la categoría de surtimiento muy bajo $(\leq 69 \%)$.

En las unidades no incorporadas a la red de servicios del SPS también se registraron incrementos significativos entre 2005 y 2006. En este último año, 21 estados $(72.4 \%)$ presentaron porcentajes de surtimiento completo de recetas considerados como medios a
Cuadro IV

Niveles de surtimiento COMPLETO de RECETAS EN UNIDADES DE ATENCIÓN AMBULATORIA POR ESTATUS DE AFILIACIÓN A LAS REDES del SPS, Méxıco 2005-2006

\begin{tabular}{lcccc} 
Porcentaje de surtimiento & 2005 & 2006 & 2005 & 2006 \\
$90-100$ & $6(19.4)$ & $16(50.0)$ & $5(20.0)$ & $11(37.9)$ \\
\hline $80-89$ & $10(32.3)$ & $10(31.2)$ & $6(24.0)$ & $10(34.5)$ \\
\hline $70-79$ & $8(25.8)$ & $3(9.4)$ & $7(28.0)$ & $4(13.8)$ \\
\hline$\leq 69$ & $7(22.5)$ & $3(9.4)$ & $7(28.0)$ & $4(13.8)$ \\
\hline Total & $31^{*}$ & 32 & $25^{\ddagger}$ & $29^{\ddagger}$
\end{tabular}

* En la medición 2005 el DF no tenía unidades incorporadas al SPS

‡ En 2005 hubo siete entidades con unidades de salud no incorporadas y en 2006 la cifra se redujo a tres

SPS: Seguro Popular de Salud

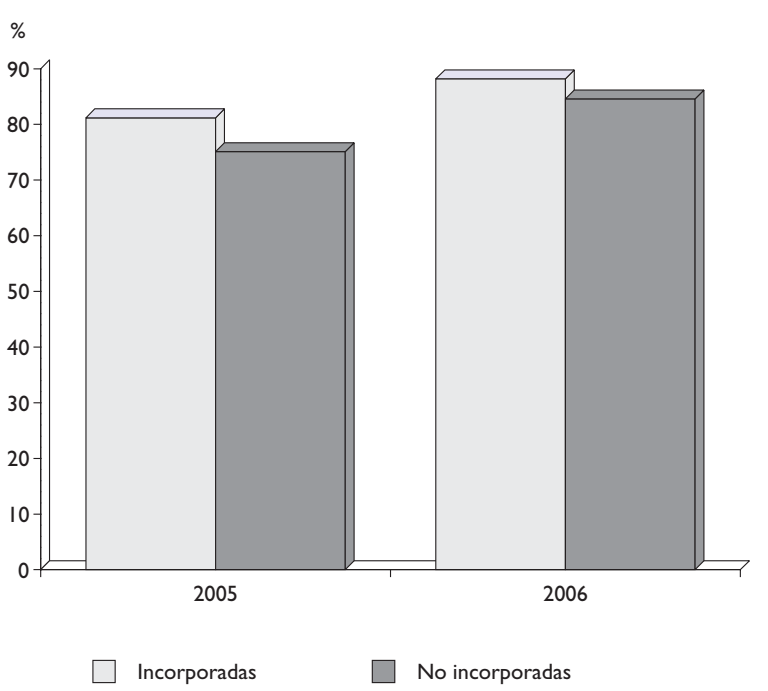

Figura 3. Porcentaje de recetas surtidas completamente EN UNIDADES AMBULATORIAS POR ESTATUS DE AFILIACIÓN A LA RED DE PROVEedores DEL SPS. MÉXICo 2005-2006

altos. En este grupo de unidades repiten con buenos desempeños Tabasco, Tamaulipas, Veracruz y Yucatán. Se agregan a la lista los estados de Baja California, Baja California Sur, Chiapas y Durango, con surtimientos superiores a $90 \%$. De igual forma, repiten con muy bajo desempeño el Estado de México y Nayarit. 


\section{Surtimiento de recetas en hospitales}

Los datos de la ENSATA 2006 demostraron que la proporción de pacientes que recibió el surtimiento completo de sus recetas al momento de egresar del hospital fue de $44.4 \%$ para los afiliados al SPS y de $18.5 \%$ para los no afiliados. Estos resultados muestran una gran diferencia en el surtimiento de recetas entre los ámbitos hospitalario y ambulatorio; mientras que el acceso a los medicamentos en el primer nivel de atención está prácticamente asegurado en una gran parte de las entidades federativas, la brecha en hospitales es todavía considerable.

\section{Eficiencia en el surtimiento de recetas}

Para evaluar la eficiencia en el surtimiento, se estudió la relación que existe entre el gasto per cápita en salud en población sin seguridad social y el surtimiento completo de recetas en las unidades de los SESA. Como puede observarse en la figura 4, hay estados como Baja California, Nuevo León, Querétaro, San Luis Potosí, Tlaxcala y Guanajuato que, con un bajo gasto per cápita en salud en población sin seguridad social, alcanzan altos niveles de surtimiento completo de recetas. En contraste, Baja California Sur, Aguascalientes, Distrito Federal y Sonora, entre otros, con un gasto per cápita en población sin seguridad social alto presentan muy bajos niveles de surtimiento completo de recetas.

\section{Satisfacción de los usuarios con el surtimiento de recetas}

La satisfacción de los usuarios con el surtimiento completo de recetas debiera ser mayor mientras mayor es el porcentaje de surtimiento completo. Esta relación no se cumple en varias entidades federativas. Durango, Guerrero, Michoacán, Morelos, Sonora, y Zacatecas presentaron, en 2005, un surtimiento bajo de recetas completas y, sin embargo, los niveles de satisfacción de sus usuarios se ubicaron por arriba del promedio nacional. La situación inversa, baja satisfacción y alto surtimiento, se observó en Campeche, Guanajuato y Sinaloa. En el resto de los estados hubo congruencia entre los niveles de surtimiento y la satisfacción de sus usuarios (figura 5).

\section{Discusión}

El SPS ha mejorado el surtimiento completo de recetas en las unidades de atención ambulatoria de los SESA, como lo demuestra el mayor porcentaje de recetas completamente surtidas en los afiliados a este seguro (89\% en 2006) que en los no afiliados (79\%) y el mayor porcentaje de recetas surtidas en unidades ambulatorias afiliadas a la red de servicios del SPS que en las no afiliadas. Además, estos porcentajes aumentaron de manera importante entre 2005 y 2006.

Entre la población sin seguridad social no afiliada al SPS que hace uso de los servicios ambulatorios y en las unidades ambulatorias no afiliadas a la red de servicios del SPS, en general, también se han presentado incrementos importantes en el surtimiento completo de recetas. Este hecho seguramente se debe a que la

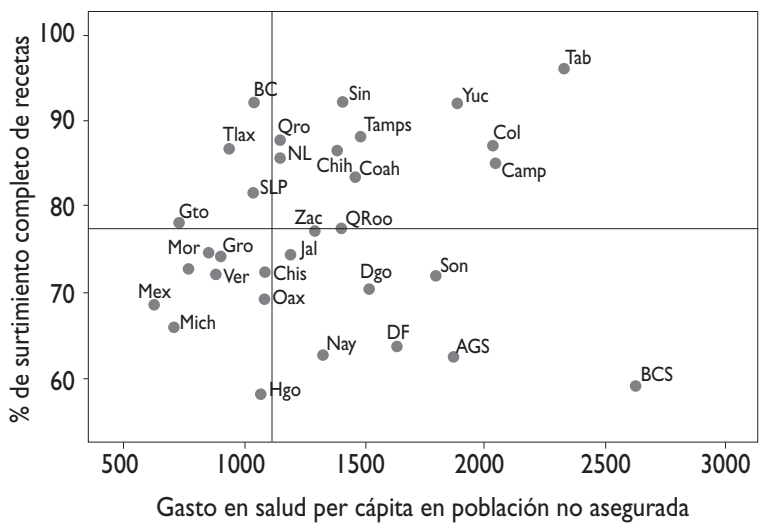

Figura 4. Gasto en salud per cápita en población Sin SEGURIDAD SOCIAL Y SURTIMIENTO COMPLETO DE RECETAS EN Los SESA POR ENTIDAD FEDERATIVA. MéXICo 2005

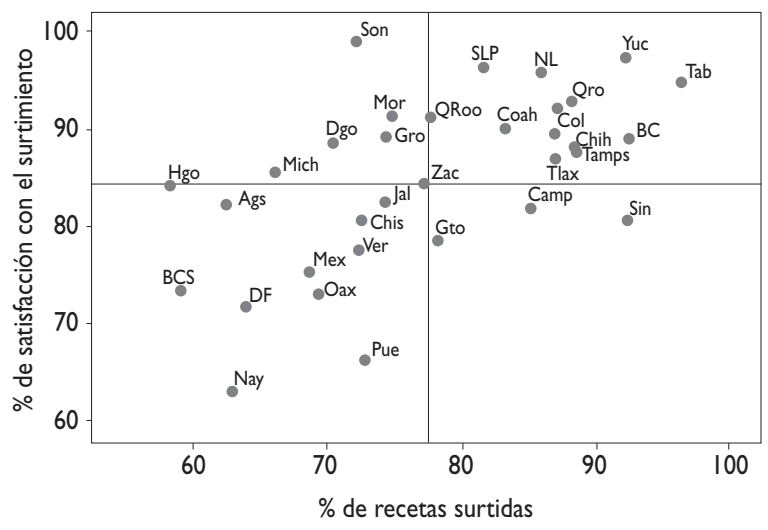

Figura 5. Surtimiento completo de RECETAS EN LOS SESA Y SATISFACCIÓN DE LOS USUARIOS CON EL SURTIMIENTO POR ENTIDAd federativa. MéXico 2005 
movilización de recursos adicionales para la compra de medicamentos y los arreglos gerenciales que mejoraron la distribución de medicamentos para los afiliados al SPS tuvieron un efecto de "derrame" sobre todas las unidades de atención ambulatoria de los SESA.

Es importante, sin embargo, señalar que los niveles actuales de surtimiento completo de recetas entre los afiliados al SPS y en las unidades ambulatorias afiliadas a la red de servicios de este seguro siguen siendo inferiores a los observados en las unidades de las instituciones de seguridad social. No obstante, los SESA de algunas entidades federativas empiezan a mostrar porcentajes de surtimiento completo de recetas en unidades ambulatorias incluso superiores a las de las instituciones de seguridad social (Baja California Sur, Campeche, Chiapas, Colima, Nuevo León, Tabasco, Tamaulipas, Tlaxcala, Veracruz, Yucatán y Zacatecas).

Cabe destacar también que, en 2006, 50\% de las entidades federativas presentaron altos niveles de surtimiento completo de recetas $(\geq 90 \%)$ entre los afiliados al SPS. Únicamente cuatro estados siguen presentando niveles de surtimiento considerados como muy bajos $(\leq 69 \%)$.

El mayor problema en el surtimiento completo de recetas a los afiliados al SPS se encuentra en los hospitales, ya que sólo $44 \%$ de los usuarios que recibieron una prescripción en los hospitales de los SESA en 2006 obtuvieron el surtimiento completo de sus recetas. Entre los no afiliados este porcentaje fue todavía más bajo $(18.5 \%)$. Este hallazgo obliga a revisar integralmente la política de medicamentos del SPS, que ha privilegiado la inclusión de claves de alta prescripción pero muy bajo costo unitario en los servicios ambulatorios, a expensas de medicamentos de costo más alto y mayor eficacia terapéutica en la atención hospitalaria, siendo estos últimos los de mayor impacto en la economía de los hogares.

El nivel de eficiencia en la provisión de medicamentos varió de manera importante entre estados. Hay entidades como Baja California, Guanajuato, San Luís Potosí y Tlaxcala que, con un bajo gasto per cápita en salud en población sin seguridad social, alcanzan altos niveles de surtimiento. En contraste, hay entidades como Aguascalientes, Baja California Sur, Distrito Federal, Durango, Jalisco, Nayarit, Sonora y Zacatecas que, con un alto gasto per cápita en salud en población sin seguridad social, presentan bajos niveles de surtimiento completo de recetas.

Por último, llaman la atención los hallazgos de la encuesta de satisfacción, con altos niveles de satisfacción en algunos estados con bajos porcentajes de surtimiento completo de recetas, y bajos niveles de satisfacción en entidades con altos porcentajes de surtimiento completo. Lo anterior puede tener varias explicaciones. En primer lugar, el concepto mismo de satisfacción es ambiguo y puede estar influido por experiencias previas de los pacientes tanto en su interacción con los servicios como en el acceso a los medicamentos: si en ocasiones anteriores los pacientes no recibían los medicamentos y ahora los reciben completa o parcialmente, las opiniones pueden ser más favorables. Por otra parte, algunos de los resultados en estados donde se iniciaron los estudios piloto del SPS, como Guanajuato y Sinaloa, donde hubo alto surtimiento y baja satisfacción, sugieren que las exigencias de la población se elevan con el tiempo y con la ampliación del seguro. Lo contrario puede estar ocurriendo en estados como Durango y Michoacán, de reciente incorporación al SPS y con alta satisfacción pero bajo surtimiento.

En estudios posteriores será necesario incorporar al análisis cifras de abasto que pueden influir en los niveles y patrones de prescripción, ya que las recetas pueden llenarse -y eventualmente surtirse de manera completa-, pero no de manera necesaria con los medicamentos requeridos, sino con aquellos disponibles. Por otra parte, también será necesario profundizar en la calidad de la prescripción

\section{Referencias}

I. Leyva-Flores R, Erviti-Erice J, Kageyama-Escobar ML,Arredondo A. Prescripción, acceso y gasto en medicamentos entre usuarios de servicios de salud en México. Salud Publica Mex 1998;40(I):24-3I.

2. Molina-Salazar R, Rivas-Vilchis JF. Over-pricing and affordability of drugs: the case of essential drugs in Mexico. Cad Saude Publica 1998; I4:50I-506. 3. Reséndez C, Garrido F, Gómez-Dantés O. Disponibilidad de medicamentos esenciales en unidades de primer nivel de la Secretaría de Salud de Tamaulipas, México. Salud Publica Mex 2000;42:298-308. 4. Gómez-Dantés O, Garrido-Latorre F,Tirado-Gómez L, Ramírez D, Macías C.Abastecimiento de medicamentos en unidades de primer nivel de atención de la Secretaría de Salud de México. Salud Publica Mex 200I;43(3):224-232.

5. Frenk J, González-Pier E, Gómez-Dantés O, Lezana MA, Knaul FM. Comprehensive reform to improve health system performance in Mexico. Lancet 2006;368: 1524-1534.

6. Secretaría de Salud. Salud: México 200I- 2005. Información para la rendición de cuentas. México, DF: Secretaría de Salud, 2006.

7. Hernández-Llamas H, Delint T, Gómez-Dantés O. Surtimiento de recetas en los Servicios Estatatales de Salud y en los Servicios de Salud del Distrito Federal. México, DF: Secretaría de Salud, 2006.

8. Sistema de Cuentas Nacionales y Estatales de Salud de la Secretaría de Salud de México. Disponible en: www.sinais.salud.gob.mx/sicuentas.html. 